\title{
Analytic and Group-Theoretic Aspects of the Cosine Transform
}

\author{
G. Ólafsson, A. Pasquale, and B. Rubin
}

\begin{abstract}
This is a brief survey of recent results by the authors devoted to one of the most important operators of integral geometry. Basic facts about the analytic family of cosine transforms on the unit sphere in $\mathbb{R}^{n}$ and the corresponding Funk transform are extended to the "higher-rank" case for functions on Stiefel and Grassmann manifolds. The main topics are the analytic continuation and the structure of polar sets, the connection with the Fourier transform on the space of rectangular matrices, inversion formulas and spectral analysis, and the group-theoretic realization as an intertwining operator between generalized principal series representations of $S L(n, \mathbb{R})$.
\end{abstract}

\section{Introduction}

The cosine transform has a long and rich history, with connections to several branches of mathematics. The name cosine transform was adopted by Lutwak [50 p. 385] for the spherical convolution which is defined on the unit sphere $\mathrm{S}^{n-1}$ in $\mathbb{R}^{n}$ by

$$
(\mathcal{C} f)(u)=\int_{\mathrm{S}^{n-1}} f(v)|u \cdot v| d v, \quad u \in \mathrm{S}^{n-1} .
$$

The motivation for this name is that the inner product $u \cdot v$ is nothing but the cosine of the angle between the unit vectors $u$ and $v$.

The following list of references shows some branches of mathematics, where the operator (1.1) and its generalizations arise in a natural way (sometimes implicitly, without naming) and play an important role.

- Convex geometry: [1, 6, 23, 24, 32, 46, 50, 69, 71, 75.

- Pseudo-Differential Operators: 15, 61.

- Group representations: [2, 3, 11, 12, 57, 60.

- Harmonic Analysis and Singular Integrals: 44, 21, 22, 27, 48, [52, 58, 59, 63, 66, 73, 74, 79.

- Integral geometry: [5, 20, 26, 30, 62, 64, 65, 68, 70, 76, 86.

- Stochastic Geometry and Probability: [29, 49, 51, 77, 78.

The authors are thankful to Tufts University for the hospitality and support during the Joint AMS meeting and the Workshop on Geometric Analysis on Euclidean and Homogeneous Spaces in January, 2012. The research of G. Ólafsson was supported by DMS-0801010 and DMS-1101337. A. Pasquale gratefully acknowledges travel support from the Commission de Colloques et Congrès Internationaux (CCCI). 


\section{- Banach Space Theory: [38, 45, 47, 54, 72].}

This list is far from being complete. In most of the publications cosine-like transforms serve as a tool for certain specific problems. At the same time, there are many papers devoted to the cosine transforms themselves. The present article is just of this kind. Our aim is to give a short overview of our recent work [57, 70] on the cosine transform and explain some of the ideas and tools behind those results.

For a complex number $\lambda$, the $\lambda$-analogue of the operator (1.1) is the convolution operator

$$
\left(\mathcal{C}^{\lambda} f\right)(u)=\int_{\mathrm{S}^{n-1}} f(v)|u \cdot v|^{\lambda} d v, \quad u \in \mathrm{S}^{n-1},
$$

where the integral is understood in the sense of analytic continuation, if necessary. We adopt the name "the cosine transform" for (1.2) too. The same name will be used for generalizations of these operators to be defined below.

In recent years more general, higher-rank cosine transforms attracted considerable attention. This class of operators was inspired by Matheron's injectivity conjecture 51, its disproval by Goodey and Howard [29, applications in group representations [7, 12, 57, 60, 86, and in algebraic integral geometry [3, [5, 20]. To the best of our knowledge, the higher-rank cosine transform was explicitly presented (without naming) for the first time in [26, formula (3.5)].

As mentioned above, the present article gives a brief survey of recent results by the authors [57, 70] in this area. The consideration grew up from specific problems of harmonic analysis and group representations. However, we do not focus on those problems, and mention them only for better explanation of the corresponding properties of the cosine transforms and related operators of integral geometry. Here we shall restrict ourselves to the case of real numbers, referring to the above articles for the case of complex and quaternionic fields.

The paper is organized as follows. Section 2 contains basic facts about the cosine transforms on the unit sphere. More general higher-rank transforms on Stiefel or Grassmann manifolds are considered in Section 3, where the main tool is the classical Fourier analysis. In Sections 4 and 5 we discuss the connections to representation theory, and more precisely to the spherical representations and the intertwining properties. Section 6 is devoted to explicit spectral formulas for the cosine transforms.

\section{Cosine transforms on the unit sphere}

In this section we discuss briefly the cosine transform on the sphere $\mathrm{S}^{n-1}$. We keep the notation from the Introduction. For the analytic continuation of the cosine transform it is convenient to normalize it by setting

$$
\left(\mathscr{C}^{\lambda} f\right)(u)=\gamma_{n}(\lambda) \int_{\mathrm{S}^{n-1}} f(v)|u \cdot v|^{\lambda} d v, \quad u \in \mathrm{S}^{n-1} .
$$

Here $d v$ stands for the $\mathrm{SO}(n)$-invariant probability measure on $\mathrm{S}^{n-1}$ and the normalizing coefficient $\gamma_{n}(\lambda)$ is given by

$$
\gamma_{n}(\lambda)=\frac{\pi^{1 / 2} \Gamma(-\lambda / 2)}{\Gamma(n / 2) \Gamma((1+\lambda) / 2)}, \quad \operatorname{Re} \lambda>-1, \quad \lambda \neq 0,2,4, \ldots
$$


This normalization is chosen so that

$$
\mathscr{C}^{\lambda}(1)=\frac{\Gamma(-\lambda / 2)}{\Gamma((n+\lambda) / 2)} .
$$

Such a normalization is convenient in many occurrences, when harmonic analysis on the sphere is performed in the multiplier language (in the same manner as analysis of pseudo-differential operators is performed in the language of their symbols). We shall see below that it also simplifies the spectrum of the cosine transform.

The limit case $\lambda=-1$ gives, up to a constant, the well-known Funk transform. Specifically, if $f \in C\left(\mathrm{~S}^{n-1}\right)$, then for every $u \in \mathrm{S}^{n-1}$,

$$
\lim _{\lambda \rightarrow-1}\left(\mathscr{C}^{\lambda} f\right)(u)=\frac{\pi^{1 / 2}}{\Gamma((n-1) / 2)}(F f)(u),
$$

where

$$
(F f)(u)=\int_{\left\{v \in S^{n-1} \mid u \cdot v=0\right\}} f(v) d_{u} v .
$$

In (2.3), $d_{u} v$ stands for the rotational invariant probability measure on the $(n-2)$ dimensional sphere $u \cdot v=0$; see, e.g., [69, Lemma 3.1].

The operators $\mathcal{C}^{\lambda}$ and $\mathscr{C}^{\lambda}$ were investigated by different approaches. A first one employs the Fourier transform technique [45, 63, 76] and relies on the equality in the sense of distributions

$$
\begin{gathered}
\left(\frac{E_{\lambda} \mathcal{C}^{\lambda} f}{\Gamma((1+\lambda) / 2)}, \mathcal{F} \omega\right)=c_{1}\left(\frac{E_{-\lambda-n} f}{\Gamma(-\lambda / 2)}, \omega\right), \\
c_{1}=2^{n+\lambda} \pi^{(n-1) / 2} \Gamma(n / 2) .
\end{gathered}
$$

Here $\omega$ is a test function belonging to the Schwartz space $S\left(\mathbb{R}^{n}\right)$,

$$
(\mathcal{F} \omega)(y)=\int_{\mathbb{R}^{n}} \omega(x) e^{i x \cdot y} d x,
$$

and $\left(E_{\lambda} f\right)(x)=|x|^{\lambda} f(x /|x|)$ denotes the extension by homogeneity.

A second approach is based on the Funk-Hecke formula, so that for each spherical harmonic $Y_{j}$ of degree $j$,

$$
\mathscr{C}^{\lambda} Y_{j}=m_{j, \lambda} Y_{j}
$$

where

$$
m_{j, \lambda}=\left\{\begin{array}{cc}
(-1)^{j / 2} \frac{\Gamma(j / 2-\lambda / 2)}{\Gamma(j / 2+(n+\lambda) / 2)} & \text { if } j \text { is even, } \\
0 & \text { if } j \text { is odd; }
\end{array}\right.
$$

see, e.g., 63. The Fourier-Laplace multiplier $\left\{m_{j, \lambda}\right\}$ forms the spectrum of $\mathscr{C}^{\lambda}$. Note that the normalizing coefficient in $\mathscr{C}^{\lambda}$ was chosen so that only factors depending on $j$ are involved in the spectral functions $\left\{m_{j, \lambda}\right\}$. The spectrum of $\mathscr{C}^{\lambda}$ encodes important information about this operator. For instance, since $m_{j, \lambda} m_{j,-\lambda-n}=1$, then for any $f \in C^{\infty}\left(\mathrm{S}^{n-1}\right)$ the following inversion formula holds:

$$
\mathscr{C}^{-\lambda-n} \mathscr{C}^{\lambda} f=f
$$

provided

$$
\lambda \in \mathbb{C}, \quad \lambda \notin\{-n,-n-2,-n-4, \ldots\} \cup\{0,2,4, \ldots\} .
$$


For the non-normalized transforms, (2.7) yields

$$
\begin{gathered}
\mathcal{C}^{-\lambda-n} \mathcal{C}^{\lambda} f=\zeta(\lambda) f, \quad \zeta(\lambda)=\frac{\Gamma^{2}(n / 2) \Gamma((1+\lambda) / 2) \Gamma((1-\lambda-n) / 2)}{\pi \Gamma(-\lambda / 2) \Gamma((n+\lambda) / 2)}, \\
\lambda \in \mathbb{C}, \quad \lambda \notin\{-1,-3,-5, \ldots\} \cup\{1-n, 3-n, 5-n, \ldots\} .
\end{gathered}
$$

Formula (2.6) reveals singularities, provides information about the kernel and the image. Moreover, it plays a crucial role in the study of cosine transforms on $L^{p}$ functions. For instance, the following statement was proved in [64, p.11], using the relevant results of Gadzhiev [21, 22 and Kryuchkov 48 for symbols of the Calderon-Zygmund singular integrals operators.

THEOREM 2.1. Let $L_{e}^{p}\left(\mathrm{~S}^{n-1}\right)$ and $L_{p, e}^{\gamma}\left(\mathrm{S}^{n-1}\right)$ be the spaces of even functions (or distributions), belonging to $L^{p}\left(\mathrm{~S}^{n-1}\right)$ and the Sobolev space $L_{p}^{\gamma}\left(\mathrm{S}^{n-1}\right)$, respectively. Then

$$
L_{p, e}^{\delta}\left(\mathrm{S}^{n-1}\right) \subset \mathscr{C}^{\lambda}\left(L_{e}^{p}\left(\mathrm{~S}^{n-1}\right)\right) \subset L_{p, e}^{\gamma}\left(\mathrm{S}^{n-1}\right)
$$

provided

$$
\begin{gathered}
\gamma=\operatorname{Re} \lambda+\frac{n+1}{2}-\left|\frac{1}{p}-\frac{1}{2}\right|(n-1), \quad \delta=\operatorname{Re} \lambda+\frac{n+1}{2}+\left|\frac{1}{p}-\frac{1}{2}\right|(n-1), \\
\lambda \notin\{0,2,4, \ldots\} \cup\{-n-1,-n-3,-n-5, \ldots\} .
\end{gathered}
$$

The embeddings (2.9) are sharp.

Finally, one can use tools from the representation theory, as we will discuss in more details in the second half of this article.

One can easily explain (2.5) - but not (2.6) - by the fact that the space of harmonic polynomials of degree $j$ is the underlying space of an irreducible representation of $K=\mathrm{SO}(n)$. Then (2.5) follows from Schur's lemma and the fact that $\mathscr{C}^{\lambda}$ commutes with rotations. Note that the group $K$ acts by the left regular representation on $L^{2}\left(S^{n-1}\right)$ and, as a representation of $K$, we have the orthogonal decomposition

$$
L^{2}\left(S^{n-1}\right) \simeq_{K} \bigoplus_{j \in \mathbb{N}_{0}} \mathcal{Y}^{j}
$$

where the set $\mathcal{Y}^{j}$ of all spherical harmonics of degree $j$ is an irreducible $K$-space. As we shall see in Section 6, the spectral multiplier (2.6) can also be computed by identifying $\mathscr{C}^{\lambda}$ as a standard intertwining operator between certain principal series representations of the larger group $\operatorname{SL}(n, \mathbb{R})$, see $[\mathbf{5 7}$.

The fact that $\mathscr{C}^{\lambda}$ is zero on the odd power harmonics follows from the observation that the kernel $|u \cdot v|^{\lambda}$ is an even function of $v$. Hence $\mathscr{C}^{\lambda}$ is actually an integral transform on the projective space $\mathrm{P}\left(\mathbb{R}^{n}\right)$, and here the analogue of (2.10) is

$$
L^{2}\left(\mathrm{P}\left(\mathbb{R}^{n}\right)\right) \simeq_{K} \bigoplus_{j \in 2 \mathbb{N}_{0}} \mathcal{Y}^{j}
$$

\section{Cosine transforms on Stiefel and Grassmann manifolds}

In this section we introduce the higher-rank cosine transforms and collect some basic facts about these transforms. The main results are presented in Theorems 3.2 , 3.3, 3.6, 3.7, and 3.8, 
3.1. Notation. We denote by $\mathrm{V}_{n, m} \sim \mathrm{O}(n) / \mathrm{O}(n-m)$ the Stiefel manifold of $n \times m$ real matrices, the columns of which are mutually orthogonal unit $n$ vectors. For $v \in \mathrm{V}_{n, m}, d v$ stands for the invariant probability measure on $\mathrm{V}_{n, m}$; $\xi=\{v\}$ denotes the linear subspace of $\mathbb{R}^{n}$ spanned by $v$. These subspaces form the Grassmann manifold $\mathrm{G}_{n, m} \sim \mathrm{O}(n) /(\mathrm{O}(n-m) \times \mathrm{O}(m))$ endowed with the invariant probability measure $d \xi$. We write $\mathrm{M}_{n, m} \sim \mathbb{R}^{n m}$ for the space of real matrices $x=\left(x_{i, j}\right)$ having $n$ rows and $m$ columns and set

$$
d x=\prod_{i=1}^{n} \prod_{j=1}^{m} d x_{i, j}, \quad|x|_{m}=\operatorname{det}\left(x^{t} x\right)^{1 / 2},
$$

$x^{t}$ being the transpose of $x$. If $n=m$, then $|x|_{m}$ is just the absolute value of the determinant of $x$; if $m=1$, then $|x|_{1}$ is the usual Euclidean norm of $x \in \mathbb{R}^{n}$.

3.2. The Cos-function. We give two equivalent "higher-rank" substitutes for $|u \cdot v|$ in (1.1). The first one is "more geometric", while the second is "more analytic". For $1 \leq m \leq k \leq n-1$, let $\eta \in \mathrm{G}_{n, m}$ and $\xi \in \mathrm{G}_{n, k}$ be linear subspaces of $\mathbb{R}^{n}$ of dimension $m$ and $k$, respectively. Following [2, 3, [57, we set

$$
\operatorname{Cos}(\xi, \eta)=\operatorname{vol}_{m}\left(\operatorname{Pr}_{\xi} E\right)
$$

where $\operatorname{vol}_{m}(\cdot)$ denotes the $m$-dimensional volume function, $E$ is a convex subset of $\eta$ of volume one containing the origin, $\operatorname{Pr}_{\xi}$ denotes the orthogonal projection onto $\xi$. By affine invariance, this definition is independent of the choice of $E$.

The second definition [31] gives precise meaning to the projection operator $\operatorname{Pr}_{\xi}$. Let $u$ and $v$ be arbitrary orthonormal bases of $\xi$ and $\eta$, respectively. We regard $u$ and $v$ as elements of the corresponding Stiefel manifolds $\mathrm{V}_{n, k}$ and $\mathrm{V}_{n, m}$. If $k=m=1$, then $u$ and $v$ are unit vectors, as in (1.1). The orthogonal projection $\operatorname{Pr}_{\xi}$ is given by the $k \times k$ matrix $u u^{t}$, and we can define

$$
\operatorname{Cos}(\xi, \eta) \equiv \operatorname{Cos}(\{u\},\{v\})=\left(\operatorname{det}\left(v^{t} u u^{t} v\right)\right)^{1 / 2} \equiv\left|u^{t} v\right|_{m} .
$$

This definition is independent of the choice of bases in $\xi$ and $\eta$ and yields $|u \cdot v|$ if $k=m=1$.

REMARK 3.1. Note that $v^{t} u u^{t} v$ is a positive semi-definite matrix, and therefore, $\operatorname{det}\left(v^{t} u u^{t} v\right) \equiv \operatorname{det}\left(u^{t} v v^{t} u\right) \geq 0$. It means that $\operatorname{Cos}(\xi, \eta)=\operatorname{Cos}(\eta, \xi) \geq 0$.

3.3. Non-normalized cosine transforms. According to (3.1) and (3.2), one can use both Stiefel and Grassmannian language in the definition of the higher-rank cosine transform, namely,

$$
\begin{gathered}
\left(\mathcal{C}_{m, k}^{\lambda} f\right)(u)=\int_{\mathrm{V}_{n, m}} f(v)\left|u^{t} v\right|_{m}^{\lambda} d v, \quad u \in \mathrm{V}_{n, k}, \\
\left(\mathcal{C}_{m, k}^{\lambda} f\right)(\xi)=\int_{\mathrm{G}_{n, m}} f(\eta) \operatorname{Cos}^{\lambda}(\xi, \eta) d \eta, \quad \xi \in G_{n, k},
\end{gathered}
$$

where $d v$ and $d \eta$ stand for the relevant invariant probability measures. The fact that we have two ways of writing of the same operator, extends the arsenal of techniques (some of them will be exhibited below). Both operators agree with $\mathcal{C}^{\lambda}$ in (1.2), when $k=m=1$. For brevity, we shall write $\mathcal{C}_{m}^{\lambda}=\mathcal{C}_{m, m}^{\lambda}$.

We remark that there are different shifts in the power $\lambda$ in the literature, all for different reasons. In particular, to make our statements in Sections 2-4 consistent with those in $\mathbf{7 0}$, one should set $\lambda=\alpha-k$. To adapt to the notation in [57] one 
has to change $\lambda$ to $\lambda-n / 2$. For unifying the presentation of the results in [70] and 57. we have preferred to adopt the unshifted notation as in (3.3) and (3.4).

Following [16, 28, the Siegel gamma function of the cone $\Omega$ of positive definite $m \times m$ real symmetric matrices is defined by

$$
\Gamma_{m}(\alpha)=\int_{\Omega} \exp (-\operatorname{tr}(r))|r|_{m}^{\alpha-(m+1) / 2} d r=\pi^{m(m-1) / 4} \prod_{j=0}^{m-1} \Gamma(\alpha-j / 2)
$$

and represents a meromorphic function with polar set

$$
\{(m-1-j) / 2 \mid j=0,1,2, \ldots\} .
$$

THEOREM 3.2. Let $1 \leq m \leq k \leq n-1$.

(i) If $f \in L^{1}\left(\mathrm{~V}_{n, m}\right)$ and $\operatorname{Re} \lambda>m-k-1$, then the integral (3.3) converges for almost all $u \in V_{n, k}$.

(ii) If $f \in C^{\infty}\left(\mathrm{V}_{n, m}\right)$, then for every $u \in V_{n, k}$, the function $\lambda \mapsto\left(\mathcal{C}_{m, k}^{\lambda} f\right)(u)$ extends to the domain $\operatorname{Re} \lambda \leq m-k-1$ as a meromorphic function with the only poles $m-k-1, m-k-2, \ldots$. These poles and their orders are the same as of the gamma function $\Gamma_{m}((\lambda+k) / 2)$.

(iii) The normalized integral $\left(\mathcal{C}_{m, k}^{\lambda} f\right)(u) / \Gamma_{m}((\lambda+k) / 2)$ is an entire function of $\lambda$ and belongs to $C^{\infty}\left(\mathrm{V}_{n, k}\right)$ in the $u$-variable.

A similar statement holds for (3.4). The proof of Theorem 3.2 can be found in [70, Theorems 4.3, 7.1]. It relies on the fact that $\left|u^{t} v\right|_{m}^{\lambda}$ is a special case of the composite power function $\left(u^{t} v\right)^{\lambda}$ with the vector-valued exponent $\lambda \in \mathbb{C}^{m}[\mathbf{1 6}, \mathbf{2 8}$. The corresponding composite cosine transforms were studied in [58, 59, $\mathbf{7 0}$.

An important ingredient of the proof of Theorem 3.2 is the connection between the cosine transform $\mathcal{C}_{m, k}^{\lambda} f$ on $\mathrm{V}_{n, m}$ and the Fourier transform

$$
\hat{\varphi}(y)=(\mathcal{F} \varphi)(y)=\int_{\mathrm{M}_{n, m}} e^{\operatorname{tr}\left(i y^{t} x\right)} \varphi(x) d x, \quad y \in \mathrm{M}_{n, m} .
$$

The corresponding Parseval equality has the form

$$
(\hat{\varphi}, \hat{\omega})=(2 \pi)^{n m}(\varphi, \omega), \quad(\varphi, \omega)=\int_{\mathrm{M}_{n, m}} \varphi(x) \overline{\omega(x)} d x .
$$

This equality with $\omega$ in the Schwartz class $S\left(\mathrm{M}_{n, m}\right)$ of smooth rapidly decreasing functions is used to define the Fourier transform of the corresponding distributions.

We will need polar coordinates on $\mathrm{M}_{n, m}$, so that for $n \geq m$, every matrix $x \in \mathrm{M}_{n, m}$ of rank $m$ can be uniquely represented as $x=v r^{1 / 2}$ with $v \in \mathrm{V}_{n, m}$ and $r=x^{t} x \in \Omega$. Given a function $f$ on $\mathrm{V}_{n, m}$, we denote $\left(E_{\lambda} f\right)(x)=|r|_{m}^{\lambda / 2} f(v)$. The following statement holds in the case $k=m$.

THEOREM 3.3. Let $f$ be an integrable right $\mathrm{O}(m)$-invariant function on $\mathrm{V}_{n, m}$, $\omega \in \mathcal{S}\left(M_{n, m}\right), 1 \leq m \leq n-1, \mathcal{C}_{m}^{\lambda} f=\mathcal{C}_{m, m}^{\lambda} f$. Then for every $\lambda \in \mathbb{C}$,

$$
\begin{gathered}
\left(\frac{E_{\lambda} \mathcal{C}_{m}^{\lambda} f}{\Gamma_{m}((\lambda+m) / 2)}, \mathcal{F} \omega\right)=c\left(\frac{E_{-\lambda-n} f}{\Gamma_{m}(-\lambda / 2)}, \omega\right), \\
c=\frac{2^{m(n+\lambda)} \pi^{n m / 2} \Gamma_{m}(n / 2)}{\Gamma_{m}(m / 2)},
\end{gathered}
$$

where both sides are understood in the sense of analytic continuation. 
The formula (3.9) agrees with (2.4). The more general statement for arbitrary $k \geq m$ can be found in $\mathbf{7 0}$.

REMARK 3.4. It is important to note that the domains, where the left-hand side and the right-hand side of of (3.9) exist as absolutely convergent integrals, have no points in common, when $m>1$. This is the principal distinction from the case $m=1$, when there is a common strip of convergence $-1<\operatorname{Re} \lambda<0$. To perform analytic continuation, we have to switch from $\mathcal{C}_{m}^{\lambda}$ to the more general composite cosine transform $\mathcal{C}_{m}^{\lambda}$ with $\lambda \in \mathbb{C}^{m}$ and then take the restriction to the diagonal $\lambda_{1}=\cdots=\lambda_{m}=\lambda+m$. This method of analytic continuation was first used by Khèkalo (for another class of operators) in his papers [39, 41, 40] on Riesz potentials on the space of rectangular matrices.

3.4. The Funk transform. The higher-rank version of the classical Funk transform (2.3) sends a function $f$ on $\mathrm{V}_{n, m}$ to a function $F_{m, k} f$ on $\mathrm{V}_{n, k}$ by the formula

$$
\left(F_{m, k} f\right)(u)=\int_{\left\{v \in \mathrm{V}_{n, m} \mid u^{t} v=0\right\}} f(v) d_{u} v, \quad u \in V_{n, k} .
$$

The condition $u^{t} v=0$ means that subspaces $\{u\} \in \mathrm{G}_{n, k}$ and $\{v\} \in \mathrm{G}_{n, m}$ are mutually orthogonal. Hence, necessarily, $k+m \leq n$. The case $k=m$, when both $f$ and its Funk transform live on the same manifold, is of particular importance and coincides with (2.3) when $k=m=1$. We denote $F_{m}=F_{m, m}$.

If $f$ is right $\mathrm{O}(m)$-invariant, $\left(F_{m, k} f\right)(u)$ can be identified with a function on the Grassmannians $\mathrm{G}_{n, m}$ or $\mathrm{G}_{n, n-m}$, and can be written "in the Grassmannian language". For instance, setting $\xi=\{v\} \in \mathrm{G}_{n, m}, \eta=\{u\}^{\perp} \in \mathrm{G}_{n, n-k}$, and $\tilde{f}(\xi)=$ $f(v)$, we obtain

$$
\left(R_{m, n-k} \tilde{f}\right)(\eta) \equiv \int_{\xi \subset \eta} \tilde{f}(\xi) d_{\eta} \xi=\left(F_{m, k} f\right)(u) .
$$

3.5. Normalized cosine transforms. Our next aim is to introduce a natural generalization $\mathscr{C}_{m, k}^{\lambda} f$ of the normalized transform (2.1). "Natural" means that we expect $\mathscr{C}_{m, k}^{\lambda} f$ to obey the relevant higher-rank modifications of the properties (2.2)(2.5).

Definition 3.5. Let $1 \leq m \leq k \leq n-1$. For $u \in V_{n, k}$ and $v \in V_{n, m}$, we define

$$
\left(\mathscr{C}_{m, k}^{\lambda} f\right)(u)=\gamma_{n, m, k}(\lambda) \int_{\mathrm{V}_{n, m}} f(v)\left|u^{t} v\right|_{m}^{\lambda} d v,
$$

where

$$
\gamma_{n, m, k}(\lambda)=\frac{\Gamma_{m}(m / 2)}{\Gamma_{m}(n / 2)} \frac{\Gamma_{m}(-\lambda / 2)}{\Gamma_{m}((\lambda+k) / 2)}, \quad \lambda+m \neq 1,2, \ldots
$$

We denote $\mathscr{C}_{m}^{\lambda}=\mathscr{C}_{m, m}^{\lambda}$. The integral (3.12) is absolutely convergent if $\operatorname{Re} \lambda>$ $m-k-1$. The excluded values of $\lambda$ belong to the polar set of $\Gamma_{m}(-\lambda / 2)$. If $k=m=1$ this definition coincides with (2.1). Operators of this kind implicitly arose in [26. pp. 367, 368].

THEOREM 3.6. Let $1 \leq m \leq k \leq n-1, k+m \leq n$. If $f$ is a $C^{\infty}$ right $\mathrm{O}(m)$-invariant function on $\mathrm{V}_{n, m}$, then for every $u \in \mathrm{V}_{n, k}$,

$$
\underset{\lambda=-k}{\operatorname{a.c.}}\left(\mathscr{C}_{m, k}^{\lambda} f\right)(u)=\frac{\Gamma_{m}(m / 2)}{\Gamma_{m}((n-k) / 2)}\left(F_{m, k} f\right)(u),
$$


where "a.c." denotes analytic continuation and $\left(F_{m, k} f\right)(u)$ is the Funk transform (3.10).

This statement follows from [70, Theorems 7.1 (iv) and 6.1]. Note that if $m=k=1$, then (3.13) yields (2.2). However, unlike (2.2), the proof of which is straightforward, 3.13 requires a certain indirect procedure, which invokes the Fourier transform on the space of matrices and the relevant analogue of (3.9).

We point out that a pointwise inversion of the Funk transform can be obtained by means of the dual cosine transform, which is defined by

$$
\left(\stackrel{\mathcal{C}}{\mathrm{C}}_{m, k}^{\lambda} \varphi\right)(v)=\int_{\mathrm{V}_{n, k}} \varphi(u)\left|u^{t} v\right|_{m}^{\lambda} d u, \quad v \in \mathrm{V}_{n, m} .
$$

Indeed, the following result holds.

Theorem 3.7. (cf. [70, Theorems 7.4]) Let $\varphi=F_{m, k} f$, where $f$ is a $C^{\infty}$ right $\mathrm{O}(m)$-invariant function on $\mathrm{V}_{n, m}, 1 \leq m \leq k \leq n-m$. Then, for every $v \in \mathrm{V}_{n, m}$,

$$
\underset{\lambda=m-n}{a . c .} \frac{\left(\stackrel{*}{\mathcal{C}}_{m, k}^{\lambda} \varphi\right)(v)}{\Gamma_{m}((\lambda+k) / 2)}=c f(v), \quad c=\frac{\Gamma_{m}(n / 2)}{\Gamma_{m}(k / 2) \Gamma_{m}(m / 2)} .
$$

Regarding other inversion methods of the higher-rank Funk transform (which is also known as the Radon transform for a pair of Grassmannnians), see [31, 85. and references therein.

In the case $k=m$ the normalized cosine transform $\mathscr{C}_{m}^{\lambda}=\mathscr{C}_{m, m}^{\lambda}$ has a number of important features. If $f \in C^{\infty}\left(\mathrm{V}_{n, m}\right)$, then analytic continuation of $\left(\mathscr{C}_{m}^{\lambda} f\right)(u)$ is well-defined for all complex $\lambda \notin\{1-m, 2-m, \ldots\}$ and belongs to $C^{\infty}\left(\mathrm{V}_{n, m}\right)$. The following inversion formulas hold.

TheOREM 3.8. (cf. [70, Theorems 7.7]) Let $f \in C^{\infty}\left(\mathrm{V}_{n, m}\right)$ be a right $\mathrm{O}(m)$ invariant function on $\mathrm{V}_{n, m}, 2 m \leq n$. Then, for every $u \in \mathrm{V}_{n, m}$,

$$
\left(\mathscr{C}_{m}^{-\lambda-n} \mathscr{C}_{m}^{\lambda} f\right)(u)=f(u), \quad \lambda,-\lambda-n \notin\{1-m, 2-m, \ldots\} .
$$

In particular, for the non-normalized transforms,

$$
\left(\mathcal{C}_{m}^{-\lambda-n} \mathcal{C}_{m}^{\lambda} f\right)(u)=\zeta(\lambda) f(u), \quad \lambda+n,-\lambda \notin\{1,2,3, \ldots\},
$$

where

$$
\zeta(\lambda)=\frac{\Gamma_{m}^{2}(n / 2) \Gamma_{m}((m+\lambda) / 2) \Gamma_{m}((m-\lambda-n) / 2)}{\Gamma_{m}^{2}(m / 2) \Gamma_{m}(-\lambda / 2) \Gamma_{m}((n+\lambda) / 2)} .
$$

Both equalities (3.16) and (3.18) are understood in the sense of analytic continuation.

In the case $m=1$, the formulas (3.16) and (3.17) coincide with (2.7) and (2.8), respectively, but the method of the proof is different.

\section{Connection to Representation Theory}

The cosine transform is closely related to the representation theory of semisimple Lie groups. In particular, as we shall now discuss, it has an important grouptheoretic interpretation as a standard intertwining operator between generalized principal series representations of $\operatorname{SL}(n, \mathbb{R})$.

In the following we shall use the notation $G=\mathrm{SL}(n, \mathbb{R}), K=\mathrm{SO}(n)$, and

$$
\left.L=\mathrm{S}(\mathrm{O}(m) \times \mathrm{O}(n-m))=\left\{\begin{array}{ll}
A & 0 \\
0 & B
\end{array}\right) \mid \begin{array}{l}
A \in \mathrm{O}(m) \\
B \in \mathrm{O}(n-m)
\end{array}, \operatorname{det}(A) \operatorname{det}(B)=1\right\}
$$


with $m \leq n-m$. Then $\mathcal{B} \equiv K / L=G_{n, m}$ is the Grassmanian of $m$-dimensional linear subspaces of $\mathbb{R}^{n}$. We fix the base point

$$
b_{o}=\left\{\left(x_{1}, \ldots, x_{m}, 0, \ldots, 0\right) \mid x_{1}, \ldots, x_{m} \in \mathbb{R}\right\} \in \mathcal{B},
$$

so that $\mathcal{B}=K \cdot b_{0}$ and every function on $\mathcal{B}$ can be regarded as a right $L$-invariant function on $K$.

From now on, our main concern is the nonnormalized cosine transform (3.3) with equal lower indices, that is, $\mathcal{C}_{m}^{\lambda} \equiv \mathcal{C}_{m, m}^{\lambda}$. We refer to [35. Chapter V] for harmonic analysis on compact symmetric spaces and [42 for the representation theory of semisimple Lie groups.

4.1. Analysis on $\mathcal{B}$ with respect to $K$. The first connection to representation theory is related to the left regular action of the group $K$ on $L^{2}(\mathcal{B})$ by

$$
(\ell(k) f)(b)=f\left(k^{-1} b\right), \quad k \in K, b \in \mathcal{B} .
$$

For an irreducible unitary representation $\left(\pi, V_{\pi}\right)$ of $K$, we consider the subspace

$$
V_{\pi}^{L}:=\left\{v \in V_{\pi} \mid \pi(k) v=v \forall k \in L\right\}, \quad L=\mathrm{S}(\mathrm{O}(m) \times \mathrm{O}(n-m)) .
$$

The representation $\left(\pi, V_{\pi}\right)$ is said to be $L$-spherical if $V_{\pi}^{L} \neq\{0\}$. As $\mathcal{B}=K / L$ is a symmetric space, the following result is a consequence of [35. Chapter IV, Lemma 3.6].

Proposition 4.1. If $\left(\pi, V_{\pi}\right)$ is L-spherical, then $\operatorname{dim} V_{\pi}^{L}=1$.

Since $V_{\pi}^{L} \neq\{0\}$, we can choose a unit vector $e_{\pi} \in V_{\pi}^{L}$. Then we define a map $\Phi_{\pi}: V_{\pi} \rightarrow C^{\infty}(\mathcal{B}) \subset L^{2}(\mathcal{B})$ by the formula

$$
\left(\Phi_{\pi} v\right)(b):=d(\pi)^{-1 / 2}\left\langle v, \pi(k) e_{\pi}\right\rangle, \quad v \in V_{\pi}, \quad b=k \cdot b_{o} \in \mathcal{B}=K \cdot b_{o},
$$

where $d(\pi)=\operatorname{dim} V_{\pi}$. This definition is meaningful because $k \cdot b_{o}=k k^{\prime} \cdot b_{o}$ for every $k^{\prime} \in L$ and $e_{\pi}$ remains fixed under the action of $\pi\left(k^{\prime}\right)$. We also set

$$
\Phi_{\pi}(v ; b):=\left(\Phi_{\pi} v\right)(b) .
$$

Recall, if $\left(\pi, V_{\pi}\right)$ and $\left(\sigma, V_{\sigma}\right)$ are two representations of a Hausdorff topological group $H$, then an intertwining operator between $\pi$ and $\sigma$ is a bounded linear operator $T: V_{\pi} \rightarrow V_{\sigma}$ such that $T \pi(h)=\sigma(h) T$ for all $h \in H$. If $\pi$ is irreducible and $T$ intertwines $\pi$ with itself, then Schur's Lemma states that $T=c$ id for some complex number $c$, 17, p. 71. The map $\Phi_{\pi}$ is a $K$-intertwining operator in the sense that it intertwines the representation $\pi$ on $V_{\pi}$ and the left regular representation $\ell$ on $L^{2}(\mathcal{B})$, so that for $b=h \cdot b_{o}$ and $k \in K$ we have

$$
\Phi_{\pi}(\pi(k) v ; b)=\left\langle\pi(k) v, \pi(h) e_{\pi}\right\rangle=\left\langle v, \pi\left(k^{-1} h\right) e_{\pi}\right\rangle=\ell(k) \Phi_{\pi}(v ; b) .
$$

Furthermore, the left regular representation $\ell$ on $L^{2}(\mathcal{B})$ is multiplicity free, see e.g. 84, Corollary 9.8.2]. Therefore, since $\left(\pi, V_{\pi}\right)$ is irreducible, any intertwining operator $V_{\pi} \rightarrow L^{2}(B)$ is by Schur's Lemma of the form $c \Phi_{\pi}$ for some $c \in \mathbb{C}$.

We let $L_{\pi}^{2}(\mathcal{B})=\operatorname{Im} \Phi_{\pi}$. Denote by $\widehat{K}_{L}$ the set of all equivalence classes of irreducible $L$-spherical representations $\left(\pi, V_{\pi}\right)$ of $K$. Then, see 35 , Chapter V, Thm. 4.3], the decomposition of $L^{2}(\mathcal{B})$ as a $K$-representation is as follows.

$$
\text { TheOREM 4.2. } L^{2}(\mathcal{B}) \simeq_{K} \bigoplus_{\pi \in \widehat{K}_{L}} L_{\pi}^{2}(\mathcal{B}) .
$$


The cosine transform is, as mentioned before, a $K$-intertwining operator, i.e., $\mathcal{C}_{m}^{\lambda}(\ell(k) f)=\ell(k) \mathcal{C}_{m}^{\lambda}(f)$ for all $k \in K$ and $f \in L^{2}(\mathcal{B})$. It follows by Schur's Lemma that for each $\pi \in \widehat{K}_{L}$ there exists a function $\eta_{\pi}$ on $\mathbb{C}$ such that

$$
\left.\mathcal{C}_{m}^{\lambda}\right|_{L_{\pi}^{2}}=\eta_{\pi}(\lambda) \text { id }
$$

Let $f \in L_{\pi}^{2}(\mathcal{B})$ of norm one. Then $\eta_{\pi}(\lambda)=\left\langle\mathcal{C}^{\lambda}(f), f\right\rangle$ and it follows that $\eta_{\pi}(\lambda)$ is meromorphic; cf. Theorem 3.2 .

4.2. Generalized spherical principal series representations of $G$. The fact that $\mathcal{C}_{m}^{\lambda}$ is a $K$-intertwining operator does not indicate how to determine the functions $\eta_{\pi}$. In the case $m=1$ and in some particular cases for the higher-rank cosine transforms [58, 59 explicit expression for $\eta_{\pi}$ can be obtained using the Funk-Hecke Theorem or the Fourier transform technique. It is a challenging open problem to proceed the same way in the most general case, using, e.g., the relevant results of Gelbart, Strichartz, and Ton-That, see, e.g., [25, 80, 82. Below we suggest an alternative way and proceed as follows.

To find $\eta_{\pi}$ explicitly, we observe that the cosine transform is an intertwining operator between certain generalized principal series representations $\left(\pi_{\lambda}, L^{2}(\mathcal{B})\right)$ of $G=\operatorname{SL}(n, \mathbb{R})$ induced from a maximal parabolic subgroup of $G$. We can then use the bigger group $G$, or better its Lie algebra, to move between $K$-types. We invoke the spectrum generating technique introduced in $[\mathbf{7}$ to build up a recursion relation between the spectral functions $\eta_{\pi}$. This finally allows us to determine all of them by knowing $\eta_{\text {trivial }}$.

The group $G=\operatorname{SL}(n, \mathbb{R})$ acts on $\mathcal{B}$ by

$$
g \cdot \eta:=\{g v \mid v \in \eta\}
$$

where $g v$ denotes the usual matrix multiplication. This action is transitive, as the $K$-action is already transitive. The stabilizer of $b_{o}$ is the group

$$
\begin{aligned}
P & =\left\{\left(\begin{array}{cc}
A & X \\
0 & B
\end{array}\right) \mid X \in \mathrm{M}_{m, n-m}, \begin{array}{l}
A \in \mathrm{GL}(m, \mathbb{R}) \\
B \in \mathrm{GL}(n-m, \mathbb{R})
\end{array} \quad \text { and } \operatorname{det}(A) \operatorname{det}(B)=1\right\} \\
& \simeq \mathrm{S}(\mathrm{GL}(m) \times \mathrm{GL}(n-m)) \ltimes \mathrm{M}_{m, n-m},
\end{aligned}
$$

where $M_{n, m}$ is the space of $n \times m$ real matrices; see Section 3.1. We then have $\mathcal{B}=G / P$.

The $K$-invariant probability measure on $\mathcal{B}$ is not $G$-invariant. But there exists a function $j: G \times \mathcal{B} \rightarrow \mathbb{R}^{+}$such that for all $f \in L^{1}(\mathcal{B})$ we have

$$
\int_{\mathcal{B}} f(b) d b=\int_{\mathcal{B}} f(g \cdot b) j(g, b)^{n} d b, \quad g \in G, \quad b \in \mathcal{B} .
$$

We include the power $n$ to adapt our notation to [57. By the associativity of the action we have $j\left(g g^{\prime}, b\right)=j\left(g, g^{\prime} \cdot b\right) j\left(g^{\prime}, b\right)$ for all $g \in G$ and $b \in \mathcal{B}$. Hence, for each $\lambda \in \mathbb{C}$ we can define a continuous representation $\pi_{\lambda}$ of $G$ on $L^{2}(\mathcal{B})$ by

$$
\left[\pi_{\lambda}(g) f\right](b):=j\left(g^{-1}, b\right)^{\lambda+n / 2} f\left(g^{-1} \cdot b\right), \quad g \in G, \quad f \in L^{2}(\mathcal{B}), \quad \beta \in \mathcal{B} .
$$

A simple change of variables shows that

$$
\left\langle\pi_{\lambda}(g) f, h\right\rangle_{L^{2}}=\left\langle f, \pi_{-\bar{\lambda}}\left(g^{-1}\right) h\right\rangle_{L^{2}}, \quad g \in G, \quad f, h \in L^{2}(\mathcal{B}) .
$$

In particular, $\pi_{\lambda}$ is unitary if and only if $\lambda$ is purely imaginary. The representations $\pi_{\lambda}$ are the so-called generalized (spherical) principal series representations (induced 
from the maximal parabolic subgroup $P$ ), in the compact picture. See e.g. [42], p. 169.

The representations $\pi_{\lambda}$ can also be realized on Stiefel manifolds as follows. According to [70, Section 7.4.3], we introduce the radial and angular components of a matrix $x \in M_{n, m}$ of rank $m$ by

$$
\operatorname{rad}(x)=\left(x^{t} x\right)^{1 / 2} \in \Omega, \quad \operatorname{ang}(x)=x\left(x^{t} x\right)^{-1 / 2} \in \mathrm{V}_{n, m},
$$

so that $x=\operatorname{ang}(x) \operatorname{rad}(x)$. Given $\lambda \in \mathbb{C}$, we define a mapping which assigns to every $g \in \mathrm{GL}(n, \mathbb{R})$ an operator $\pi_{\lambda}(g)$ acting on measurable functions $f$ on $\mathrm{V}_{n, m}$ by the rule

$$
\pi_{\lambda}(g) f(v)=\left|\operatorname{rad}\left(g^{-1} v\right)\right|^{-(\lambda+n / 2)} f\left(\operatorname{ang}\left(g^{-1} v\right)\right) .
$$

Clearly, $\pi_{\lambda}\left(I_{n}\right)$ is an identity operator. One can prove that if $f$ is a measurable right $\mathrm{O}(m)$-invariant function on $\mathrm{V}_{n, m}$, then

$$
\pi_{\lambda}\left(g_{1} g_{2}\right) f=\pi_{\lambda}\left(g_{1}\right) \pi_{\lambda}\left(g_{2}\right) f, \quad g_{1}, g_{2} \in \mathrm{GL}(n, \mathbb{R}) .
$$

The restriction of $\pi_{\lambda}$ to $\operatorname{SL}(n, \mathbb{R})$, acting on the space of square integrable right $\mathrm{O}(m)$-invariant functions on $\mathrm{V}_{n, m}$, coincides with the representation defined by (4.4).

4.3. The cosine transform as an intertwining operator. In this section we follow the ideas in [57. An alternative self-contained exposition (without using the representation theory of semisimple Lie groups), can be found in $\mathbf{7 0}$.

The gain by using the representations $\pi_{\lambda}$ is that we now have a meromorphic family of representations on $L^{2}(\mathcal{B})$ and that they are irreducible for almost all $\lambda$ and closely related to the cosine transform. For this, we recall some results from 83.

Theorem 4.3 (Vogan-Wallach). There exists a countable collection $\left\{p_{n}\right\}$ of non-zero holomorphic polynomials on $\mathbb{C}$ such that if $p_{n}(\lambda) \neq 0$ for all $n$ then $\pi_{\lambda}$ is irreducible. In particular, $\pi_{\lambda}$ is irreducible for almost all $\lambda \in \mathbb{C}$.

Proof. This is Lemma 5.3 in $\mathbf{8 3}$.

Let $\theta: G \rightarrow G$ be the involutive automorphism $\theta(g)=\left(g^{-1}\right)^{t}$. We remark that in 57. notation $\operatorname{Cos}^{\lambda}=\mathcal{C}_{m}^{\lambda-n / 2}$ was used.

THEOREM 4.4. The cosine transform intertwines $\pi_{\lambda}$ and $\pi_{-\lambda} \circ \theta$, namely,

$$
\mathcal{C}_{m}^{\lambda} \circ \pi_{\lambda+n / 2}=\left(\pi_{-\lambda-n / 2} \circ \theta\right) \circ \mathcal{C}_{m}^{\lambda},
$$

whenever both sides of this equality are analytic functions of $\lambda$.

Proof. We refer to Theorem 2.3 and (4.10) in [57.

In fact, it is shown in [57, Lemma 2.5 and Theorem 4.2 , that $\mathcal{C}_{m}^{\lambda-n / 2}=J(\lambda)$, where $J(\lambda)$ is a standard intertwining operator, studied in detail among others by Knapp and Stein in [43, 44] and Vogan and Wallach in 83. These authors show, in particular, that $\lambda \mapsto J(\lambda)$ has a meromorphic extension to all of $\mathbb{C}$. Furthermore, Vogan and Wallach show that if $f \in C^{\infty}(\mathcal{B})$, then the map

$$
\{\lambda \in \mathbb{C} \mid \operatorname{Re}(\lambda)>-1+n / 2\} \ni \lambda \longmapsto J(\lambda) f \in C^{\infty}(\mathcal{B})
$$

is holomorphic. As a consequence of $\mathcal{C}_{m}^{\lambda-n / 2}=J(\lambda)$ and [83, $\left.1.6 \mathrm{Thm}\right]$, we get the following theorem. 
TheOREM 4.5. The map $\lambda \mapsto \mathcal{C}_{m}^{\lambda}$ extends meromorphically to $\mathbb{C}$. In particular, for $f \in C^{\infty}(\mathcal{B})$ the function $\lambda \mapsto \mathcal{C}_{m}^{\lambda}(f)(b)$ extends to a meromorphic function on $\mathbb{C}$ and the set of possibles poles can be chosen independent of $f$. In the complement of the singular set we have $\mathcal{C}_{m}^{\lambda}(f) \in C^{\infty}(\mathcal{B})$.

Notice that precise information about analiticity of more general cosine transforms, including the structure of polar sets, is presented in Theorem 3.2 above.

The implication of (4.7) is that $\mathcal{C}_{m}^{-\lambda-n / 2} \circ \mathcal{C}_{m}^{\lambda-n / 2}$ intertwines $\pi_{\lambda}$ with itself (in the sense of meromorphic family of operators). By Theorem 4.3 there exists a meromorphic function $\eta$ on $\mathbb{C}$ such that

$$
\mathcal{C}_{m}^{-\lambda-n / 2} \circ \mathcal{C}_{m}^{\lambda-n / 2}=\eta(\lambda) \operatorname{id}_{C^{\infty}(\mathcal{B})}
$$

for all $\lambda \in \mathbb{C}$ for which the left-hand side is well defined. The shift by $n / 2$ in the definition is chosen so that the final formulas agree with those in [57. and make some formulas more symmetric. The fact that $\eta$ is meromorphic follows by noting that $\eta(\lambda)=\left\langle\mathcal{C}_{m}^{-\lambda-n / 2} \circ \mathcal{C}_{m}^{\lambda-n / 2}(1), 1\right\rangle$.

Formula (4.8) is a symmetric version of (3.18) with $\lambda$ replaced by $\lambda-n / 2$. The explicit value of $\eta(\lambda)$ can be easily obtained from (3.17). An alternative, representation-theoretic method to compute the function $\eta(\lambda)$, is presented in Section 6. The first step is the following lemma.

$$
\text { Lemma 4.6. Let } c(\lambda)=\mathcal{C}_{m}^{\lambda-n / 2}(1) \text {. Then } \eta(\lambda)=c(\lambda) c(-\lambda) \text {. }
$$

Note that $c(\lambda)$ is nothing but $\eta_{\text {trivial }}(\lambda)$ in Theorem 6.6

REMARK 4.7. There are several ways to prove the meromorphic extension of the standard intertwining operators. The proof in 83 uses tensoring with finite dimensional representations of $G$ to deduce a relationship between $\mathcal{C}_{m}^{\lambda}$ and $\mathcal{C}_{m}^{\lambda+2 n}$. In fact, there exists a family of (non-invariant) differential operators $D_{\lambda}$ on $\mathcal{B}$ and a polynomial $b(\lambda)$, the Bernstein polynomial, such that

$$
b(\lambda) \mathcal{C}_{m}^{\lambda}(f)=\mathcal{C}_{m}^{\lambda+2 n}\left(D_{\lambda}(f)\right)
$$

83. Thm. 1.4]. Another way to derive an equation of the form (4.9) is to convert the integral defining $\mathcal{C}_{m}^{\lambda}$ into an integral over the orbit of certain nilpotent group $\bar{N}$, as usually done in the study of standard intertwining operators, and then use the ideas from $[\mathbf{8}, \mathbf{5 5}, \mathbf{5 6}$. In the case where $G / P$ is a symmetric $R$-space (which contains the case of Grassmann manifolds), the standard intertwining operators $J(\lambda)$ have been recently studied by Clerc in [9], using Loos' theory of positive Jordan triple systems. In particular, Clerc explicitly computes the Bernstein polynomials $b(\lambda)$ in (4.9), and, hence, proves the meromorphic extension of $J(\lambda)$ for this class of symmetric spaces.

Finally, one can stick with the domain where $\lambda \mapsto \mathcal{C}_{m}^{\lambda}$ is holomorphic and determine the $K$-spectrum functions $\eta_{\pi}(\lambda)$ in (4.2). As rational functions of $\Gamma$ factors, these functions have meromorphic extension to $\mathbb{C}$. Hence, $\lambda \mapsto \mathcal{C}_{m}^{\lambda}$ itself has meromorphic extension by (4.2). We will comment more on that in Remark 6.8 .

4.4. Historical remarks. We conclude this section with a few historical remarks. The standard intertwining operators $J(\lambda)$, as a meromorphic family of singular integral operators on $K$ or $\bar{N}$, have been central objects in the study of 
representation theory of semimisimple Lie groups since the fundamental works of Knapp and Stein [43, 44, Harish-Chandra [33, and several others. In our case

$$
\bar{N}=\left\{\left(\begin{array}{cc}
I_{m} & 0 \\
X & I_{n-m}
\end{array}\right) \mid X \in \mathrm{M}_{m, n-m}\right\} .
$$

Then, in the realization of the generalized principal series representations on $L^{2}(\mathcal{B})$, the kernel of $J(\lambda)$ is $\operatorname{Cos}^{\lambda-n / 2}(b, c)$. But in most cases there is neither an explicit formula nor geometric interpretation of the kernel defining $J(\lambda)$.

Apart of customary applications of the cosine transform in convex geometry, probability, and the Banach space theory, similar integrals turned up independently as standard intertwining operators between generalized principal series representations of $\mathrm{SL}(n, \mathbb{K})$, where $\mathbb{K}=\mathbb{R}, \mathbb{C}$ or $\mathbb{H}$.

The real case was studied in [12], the complex case in [14, and the quaternionic case in [60]. In these articles it was shown that integrals of the form

$$
\int_{\mathcal{B}}|(x, y)|^{\lambda-n / 2} f(x) d x,
$$

with some modification for $\mathbb{K}=\mathbb{C}$ or $\mathbb{H}$, define intertwining operators between generalized principal series representations induced from a maximal parabolic subgroup in $\mathrm{SL}(n+1, \mathbb{K})$. The $K$-spectrum was determined, yielding the cases of irreducibility and, more generally, the composition series of those representations. Among the applications, there were some embeddings of the complementary series and the study of the so-called canonical representations on some Riemannian symmetric spaces of the noncompact type, 10, 11, 13. However the connections of these considerations to convex geometry, to the cosine transform and to the Funk and Radon transforms was neither discussed nor mentioned. These connections were first published in [57 in the context of the Grassmannians over $\mathbb{R}, \mathbb{C}$ and $\mathbb{H}$. However, it was probably S. Alesker who was the first to remark in the unpublished manuscript $[2$ that the cosine transform is a $\mathrm{SL}(n, \mathbb{R})$-intertwining operator. It was also shown in $8 \mathbf{8 6}$ that the $\operatorname{Sin}^{\lambda}$-transform (a transform related to the sine transform) can be viewed as a Knapp-Stein intertwining operator. This was used to construct complementary series representations for $\operatorname{GL}(2 n, \mathbb{R})$. The $\operatorname{Sin}^{\lambda}$ - transform is then also naturally linked to reflection positivity, which relates complementary series representations of $\mathrm{GL}(2 n, \mathbb{R})$ to the highest weight representations of $\mathrm{SU}(n, n)$, 18, 19, 53, 37, 36. Notice, however, that the definition of the $\operatorname{Sin}^{\lambda}$-transform in [86] differs from the one in [66, [70]; see also 67] for the sine transform on the hyperbolic space.

\section{The spherical representations}

The functions $\eta_{\pi}(\lambda)$ in (4.2) are parametrized by the $L$-spherical representations of $K$. The main purpose of this section is to present this parametrization, which is given by a semilattice in a finite dimensional Euclidean space associated with a maximal flat submanifold of $\mathcal{B}$. We will, therefore, have to study the structure of the symmetric space $\mathcal{B}$. We refer to [81] and the books by Helgason [34, 35, for more detailed discussions and proofs. To bring the discussion closer to standard references in Lie theory we also introduce some Lie theoretical notation which we have avoided so far. 
Let

$$
\begin{aligned}
\mathfrak{g} & =\left\{X \in \mathrm{M}_{n, n} \mid \operatorname{tr}(X)=0\right\}, \\
\mathfrak{k} & =\left\{X \in \mathrm{M}_{n, n} \mid X^{t}=-X\right\},
\end{aligned}
$$

be the Lie algebras of $G=\mathrm{SL}(n, \mathbb{R})$ and $K=\mathrm{SO}(n)$, respectively. The derived involution of $\theta$ on $\mathfrak{g}$, still denoted $\theta$, is given by $\theta(X)=-X^{t}$. Hence, $\mathfrak{k}=\mathfrak{g}(1, \theta)$, the eigenspace of $\theta$ on $\mathfrak{g}$ with eigenvalue 1 . We fix once and for all the $G$-invariant bilinear form $\beta(X, Y)=\frac{n}{m(n-m)} \operatorname{tr}(X Y)$ on $\mathfrak{g}$. Note that $\beta$ is negative definite on $\mathfrak{k}$ and $\langle X, Y\rangle=-\beta(X, \theta(Y))$ is an inner product on $\mathfrak{g}$ such that $\operatorname{ad}(X)^{t}=-\operatorname{ad}(\theta(X))$, where, as usual, $\operatorname{ad}(X) Y=[X, Y]=X Y-Y X$. The normalization of $\beta$ is chosen so that it agrees with [57].

We recall that $\mathcal{B}$ is a symmetric space corresponding to the involution

$$
\tau(x)=\left(\begin{array}{cc}
\mathrm{I}_{m} & 0 \\
0 & -\mathrm{I}_{n-m}
\end{array}\right) x\left(\begin{array}{cc}
\mathrm{I}_{m} & 0 \\
0 & -\mathrm{I}_{n-m}
\end{array}\right)=\left(\begin{array}{cc}
A & -B \\
-C & D
\end{array}\right) \quad \text { for } \quad x=\left(\begin{array}{cc}
A & B \\
C & D
\end{array}\right),
$$

where for $r \in \mathbb{N}$ we denote by $\mathrm{I}_{r}$ the $r \times r$ identity matrix. Note that $\tau$ in fact defines an involution of $G$ and that the derived involution on the Lie algebra $\mathfrak{g}$ is given by the same form.

We have $\mathfrak{k}=\mathfrak{l} \oplus \mathfrak{q}$ where $\mathfrak{l} \simeq \mathfrak{s o}(m) \times \mathfrak{s o}(n-m)$ is the Lie algebra of $L$ and

$$
\mathfrak{q}=\mathfrak{k}(-1, \tau)=\left\{Q(X)=\left(\begin{array}{cc}
0_{m m} & X \\
-X^{t} & 0_{n-m, n-m}
\end{array}\right) \mid X \in \mathrm{M}_{m, n-m}\right\} .
$$

Let $E_{\nu, \mu}=\left(\delta_{i \nu} \delta_{j \mu}\right)_{i, j}$ denote the matrix in $\mathrm{M}_{m, n-m}$ with all entries equal to 0 but the $(\nu, \mu)$-th which is equal to 1 . For $\mathbf{t}=\left(t_{1}, \ldots, t_{m}\right)^{t} \in \mathbb{R}^{m}$ we set

$$
\begin{aligned}
X(\mathbf{t}) & =-\sum_{j=1}^{m} t_{j} E_{n-2 m+j, j} \in \mathrm{M}_{m, n-m}, \\
Y(\mathbf{t}) & =Q(X(\mathbf{t})) \in \mathfrak{q} .
\end{aligned}
$$

Then $\mathfrak{b}=\left\{Y(\mathbf{t}) \mid \mathbf{t} \in \mathbb{R}^{m}\right\} \simeq \mathbb{R}^{m}$ is a maximal abelian subspace of $\mathfrak{q}$.

To describe the set $\widehat{K}_{L}$ we note first that $\mathcal{B}$ is not simply connected. So we cannot use the Cartan-Helgason theorem [35. p. 535] directly, but only a slight modification is needed. Define $\epsilon_{j}(Y(\mathbf{t})):=i t_{j}$. We will identify the element $\lambda=$ $\sum_{j=1}^{m} \lambda_{j} \epsilon_{j} \in \mathfrak{b}_{\mathbb{C}}^{*}$ with the corresponding vector $\lambda=\left(\lambda_{1}, \ldots, \lambda_{m}\right)$.

If $H \in \mathfrak{b}$, then $\operatorname{ad}(H)$ is skew-symmetric on $\mathfrak{k}$ with respect to the inner product $\langle\cdot, \cdot\rangle$. Hence $\operatorname{ad}(H)$ is diagonalizable over $\mathbb{C}$ with purely imaginary eigenvalues. For $\alpha \in i \mathfrak{b}^{*}$ let

$$
\mathfrak{k}_{\mathbb{C}}^{\alpha}:=\left\{X \in \mathfrak{k}_{\mathbb{C}} \mid(\forall H \in \mathfrak{b}) \operatorname{ad}(H) X=\alpha(H) X\right\}
$$

be the joint $\alpha$-eigenspace. Let

$$
\Delta_{\mathfrak{k}}=\left\{\alpha \in i \mathfrak{b}^{*} \mid \alpha \neq 0 \quad \text { and } \quad \mathfrak{k}_{\mathbb{C}}^{\alpha} \neq\{0\}\right\} .
$$

The dimension of $\mathfrak{k}_{\mathbb{C}}^{\alpha}$ is called the multiplicity of $\alpha$ (in $\mathfrak{k}_{\mathbb{C}}$ ).

LEMMA 5.1. We have

$$
\Delta_{\mathfrak{k}}=\left\{ \pm \epsilon_{i} \pm \epsilon_{j}(1 \leq i \neq j \leq m, \pm \text { independently }), \pm \epsilon_{i}(1 \leq i \leq m)\right\}
$$

with multiplicities respectively 1 (and not there if $m=1$ ), $2 n-m$ (and not there if $m=n-m)$. 
Proof. This follows from [34]: the table on page 518, the description of the simple root systems on page $462 \mathrm{ff}$. and the Satake diagrams on pages 532-533.

We let

$$
\Delta_{\mathfrak{k}}^{+}=\left\{\epsilon_{i} \pm \epsilon_{j}(1 \leq i<j \leq m), \epsilon_{i}(1 \leq i \leq m)\right\} .
$$

Lemma 5.2. Let $\rho_{\mathfrak{k}}=\frac{1}{2} \sum_{\alpha \in \Delta_{\mathfrak{k}}^{+}} \operatorname{dim}\left(\mathfrak{k}_{\mathbb{C}}^{\alpha}\right) \alpha \in i \mathfrak{b}^{*}$. Then $\rho_{\mathfrak{k}}=\sum_{j=1}^{m}\left(\frac{n}{2}-j\right) \epsilon_{j}$.

Let now $\left(\pi, V_{\pi}\right)$ be a unitary irreducible representation of $K$. Then $V_{\pi}$ is finite dimensional. Moreover, $\pi(H)=\left.\frac{d}{d t}\right|_{t=0} \pi(\exp (t H))$ is skew-symmetric, hence, diagonalizable, for all $H \in \mathfrak{b}$ (in fact, $\pi(H)$ is diagonalizable for all $H \in \mathfrak{k}$ ). Let $\Gamma(\pi) \subset i \mathfrak{b}^{*}$ be the finite set of joint eigenvalues of $\pi(H)$ with $H \in \mathfrak{b}$. For $\mu \in \Gamma(\pi)$, let $V_{\pi}^{\mu} \subset V_{\pi}$ denote the joint eigenspace of eigenvalue $\mu$. If $X \in \mathfrak{k}_{\mathbb{C}}^{\alpha}$ and $v \in V_{\pi}^{\mu}$, then $\pi(X) v \in V_{\pi}^{\mu+\alpha}$. Thus, there exists a $\mu=\mu_{\pi} \in \Gamma(\pi)$ such that $\pi\left(\mathfrak{k}_{\mathbb{C}}^{\alpha}\right) V_{\pi}^{\mu}=\{0\}$ for all $\alpha \in \Delta_{\mathfrak{k}}^{+}$. This only uses that $\pi$ is finite dimensional, but the irreducibility implies that this $\mu$ is unique. It is called the highest weight of $\pi$. Finally we have $\pi \simeq \sigma$ if and only if $\mu_{\pi}=\mu_{\sigma}$.

Let $\widetilde{K}$ be the universal covering group of $K$. Then $\tau$ lifts to an involution $\widetilde{\tau}$ on $\widetilde{K}, \widetilde{L}:=\widetilde{K} \widetilde{\tau}$ is connected, and $\widetilde{\mathcal{B}}:=\widetilde{K} / \widetilde{L}$ is the universal covering of $\mathcal{B}$. Replacing $K$ by $\widetilde{K}$ etc., we can talk about $\widetilde{L}$-spherical representations of $\widetilde{K}$ and their highest weights. The following theorem is a consequence of the Cartan-Helgason theorem [35, p. 535].

TheOREM 5.3. The map $\pi \mapsto \mu_{\pi}$ sets up a bijection between the set of $\widetilde{L}$ spherical representations of $\widetilde{K}$ and the semi-lattice

$$
\Lambda^{+}(\widetilde{\mathcal{B}})=\left\{\mu \in i \mathfrak{b}^{*} \mid\left(\forall \alpha \in \Delta_{\mathfrak{k}}^{+}\right) \frac{\langle\mu, \alpha\rangle}{\langle\alpha, \alpha\rangle} \in \mathbb{Z}^{+}\right\} .
$$

Furthermore, if $m=n / 2$, then

$$
\Lambda^{+}(\widetilde{\mathcal{B}})=\left\{\left(\mu_{1}, \ldots, \mu_{m}\right) \in \mathbb{Z}^{m}\left|\mu_{1} \geq \mu_{2} \geq \cdots \geq \mu_{m-1} \geq\right| \mu_{m} \mid\right\} .
$$

Otherwise,

$$
\Lambda^{+}(\widetilde{\mathcal{B}})=\left\{\left(\mu_{1}, \ldots, \mu_{m}\right) \in \mathbb{Z}^{m} \mid \mu_{1} \geq \mu_{2} \geq \cdots \geq \mu_{m-1} \geq \mu_{m} \geq 0\right\} .
$$

If $\mu \in \Lambda^{+}(\widetilde{\mathcal{B}})$, then we write $\left(\pi_{\mu}, V_{\mu}\right)$ for the corresponding $\widetilde{L}$-spherical representation. Recall the notation $\Phi_{\pi_{\mu}}$ from (4.1). Let $\Lambda^{+}(\mathcal{B})$ denote the sublattice in $\Lambda^{+}(\widetilde{\mathcal{B}})$ which corresponds to $L$-spherical representations of $K$. Then $\mu \in \Lambda^{+}(\mathcal{B})$ if and only if the functions $\Phi_{\pi_{\mu}}(v)$, which are originally defined on $\widetilde{\mathcal{B}}$, factor to functions on $\mathcal{B}$. For that, let $v \in V_{\mu}^{\mu}$ and $H \in \mathfrak{b}$. We can normalize $v$ and $e_{\pi_{\mu}}$ so that

$$
\Phi_{\pi_{\mu}}(v ; \exp H)=e^{\mu(H)} .
$$

The same argument as for the sphere [81, Ch. III.12], proves the following theorem.

THeOREM 5.4. If $m=n-m$, then

$$
\Lambda^{+}(\mathcal{B})=\left\{\mu=\sum_{j=1}^{m} \mu_{j} \varepsilon_{j} \mid \mu_{j} \in 2 \mathbb{N}_{0} \quad \text { and } \quad \mu_{1} \geq \ldots \geq \mu_{m-1} \geq\left|\mu_{m}\right|\right\} .
$$


In all other cases,

$$
\Lambda^{+}(\mathcal{B})=\left\{\mu=\sum_{j=1}^{m} \mu_{j} \varepsilon_{j} \mid \mu_{j} \in 2 \mathbb{N}_{0} \quad \text { and } \quad \mu_{1} \geq \ldots \geq \mu_{m} \geq 0\right\} .
$$

\section{The generation of the $K$-spectrum}

Recall from Section 5 the involution $\theta(X)=-X^{t}$ on $\mathfrak{g}$. The Lie algebra $\mathfrak{g}$ decomposes into eigenspaces of $\theta$ as $\mathfrak{g}=\mathfrak{k} \oplus \mathfrak{s}$, where

$$
\mathfrak{s}=\mathfrak{g}(-1, \theta)=\left\{X \in \mathrm{M}_{n, n} \mid \theta(X)=-X \text { and } \operatorname{Tr}(X)=0\right\} .
$$

Then, except in the case $n=2$, the complexification $\mathfrak{s}_{\mathbb{C}}$ of $\mathfrak{s}$ is an irreducible $L$ spherical representation of $K$. For $n=2$ this representation decomposes into two one-dimensional representations.

Let

$$
H_{o}=\left(\begin{array}{cc}
\frac{n-m}{n} \mathrm{I}_{m} & 0 \\
0 & -\frac{m}{n} \mathrm{I}_{n-m}
\end{array}\right) \in \mathfrak{s} .
$$

Then $H_{o}$ is $L$-fixed and $\left\langle H_{0}, H_{0}\right\rangle=1$. Define $\mathfrak{a}:=\mathbb{R} H_{o}$. The operator $\operatorname{ad}\left(H_{0}\right)$ has spectrum $\{0,1,-1\}$ and $\mathfrak{n}=\mathfrak{g}\left(1, \operatorname{ad}\left(H_{0}\right)\right)$.

Let $\operatorname{Ad}(k)$ denote the conjugation by $k$. Define a map $\omega: \mathfrak{s}_{\mathbb{C}} \rightarrow C^{\infty}(\mathcal{B})$ by

$$
\omega(Y)(k):=\left\langle Y, \operatorname{Ad}(k) H_{o}\right\rangle=\beta\left(Y, \operatorname{Ad}(k) H_{o}\right)=\frac{n}{m(n-m)} \operatorname{Tr}\left(Y k H_{o} k^{-1}\right)
$$

and note that

$$
\omega(\operatorname{Ad}(h) Y)(k)=\left\langle\operatorname{Ad}(h) Y, \operatorname{Ad}(k) H_{o}\right\rangle=\left\langle Y, \operatorname{Ad}\left(h^{-1} k\right) H_{o}\right\rangle=\omega(Y)\left(h^{-1} k\right) .
$$

Thus $\omega$ is a $K$-intertwining operator.

Fix an orthonormal basis $X_{1}, \ldots, X_{\operatorname{dim} \mathfrak{q}}$ of $\mathfrak{q}$ such that $X_{1}, \ldots, X_{m}$, is an orthonormal basis of $\mathfrak{b}$. Denote by $\Omega=-\sum_{j} X_{j}^{2}$ the corresponding positive definite Laplace operator on $\mathcal{B}$. Then

$$
\left.\Omega\right|_{L_{\mu}^{2}(\mathcal{B})}=\omega(\mu) \mathrm{id},
$$

where

$$
\omega(\mu)=\left\langle\mu+2 \rho_{\mathfrak{k}}, \mu\right\rangle .
$$

A simple calculation then gives:

Lemma 6.1. Let $\mu=\left(\mu_{1}, \ldots, \mu_{m}\right) \in \Lambda^{+}(\mathcal{B})$. Then

$$
\omega(\mu)=\frac{m(n-m)}{2 n} \sum_{j=1}^{m}\left(\mu_{j}^{2}+\mu_{j}(n-2 j)\right) .
$$

For $f \in C^{\infty}(\mathcal{B})$ denote by $M(f): L^{2}(\mathcal{B}) \rightarrow L^{2}(\mathcal{B})$ the multiplication operator $g \mapsto f g$. Recall the notation $\pi_{0}$ for the finite dimensional spherical representation of highest weight $0 \in \Lambda^{+}(\mathcal{B})$.

Theorem 6.2. Let $Y \in \mathfrak{s}$. Then $[\Omega, M(\omega(Y))]=2 \pi_{0}(Y)$.

Proof. This is Theorem 2.3 in [7].

For $\mu \in \Lambda^{+}(\mathcal{B})$ define $\Psi_{\mu}: L_{\mu}^{2}(\mathcal{B}) \otimes \mathfrak{s}_{\mathbb{C}} \rightarrow L^{2}(\mathcal{B})$ by

$$
\Psi_{\mu}(\varphi \otimes Y):=M(\omega(Y)) \varphi \text {. }
$$

Observe that for $k \in K, Y \in \mathfrak{s}_{\mathbb{C}}$, and $\varphi \in L_{\mu}^{2}(\mathcal{B})$ we have

$$
\ell(k)(M(\omega(Y) \varphi)=(\ell(k) \omega(Y))(\ell(k) \varphi)=M(\omega(\operatorname{Ad}(k) Y))(\ell(k) \varphi)
$$


with $\operatorname{Ad}(k) Y \in \mathfrak{s}_{\mathbb{C}}$ and $\ell(k) \varphi \in L_{\mu}^{2}(\mathcal{B})$. Hence, $\Psi_{\mu}$ is $K$-equivariant and $\operatorname{Im} \Psi_{\mu}$ is $K$-invariant. Define a finite subset $S(\mu) \subset \Lambda^{+}(\mathcal{B})$ by

$$
\operatorname{Im} \Psi_{\mu} \simeq_{K} \bigoplus_{\sigma \in S(\mu)} L_{\sigma}^{2}(\mathcal{B}) .
$$

Lemma 6.3. Let $\mu \in \Lambda^{+}(\mathcal{B})$. Then

$$
S(\mu)=\left\{\mu \pm 2 \epsilon_{j} \mid j=1, \ldots, m\right\} \cap \Lambda^{+}(\mathcal{B}) .
$$

These representations occur with multiplicity one.

Denote by $\operatorname{pr}_{\sigma}$ the orthogonal projection $L^{2}(\mathcal{B}) \rightarrow L_{\sigma}^{2}(\mathcal{B})$. The first spectrum generating relation which follows from Theorem 6.2. see also [7, Cor. 2.6], states:

Lemma 6.4. Assume that $\mu \in \Lambda^{+}(\mathcal{B})$. Let $\sigma \in S(\mu), Y \in \mathfrak{s}_{\mathbb{C}}$, and $\lambda \in \mathbb{C}$. Let

$$
\omega_{\sigma \mu}(Y):=\left.\operatorname{pr}_{\sigma} \circ M(\omega(Y))\right|_{L_{\mu}^{2}(\mathcal{B})}: L_{\mu}^{2}(\mathcal{B}) \rightarrow L_{\sigma}^{2}(\mathcal{B}) .
$$

Then

$$
\left.\operatorname{pr}_{\sigma} \circ \pi_{\lambda}(Y)\right|_{L_{\mu}^{2}(\mathcal{B})}=\frac{1}{2}\left(\omega(\sigma)-\omega(\mu)+2 \frac{m(n-m)}{n} \lambda\right) \omega_{\sigma \mu}(Y) .
$$

The spectrum generating relation that we are looking for can now easily be deducted and we get:

Lemma 6.5. Let $\mu=\left(\mu_{1}, \ldots, \mu_{m}\right) \in \Lambda^{+}(\mathcal{B})$ and $\lambda \in \mathbb{C}$. Then

$$
\frac{\eta_{\mu+2 \epsilon_{j}}(\lambda)}{\eta_{\mu}(\lambda)}=\frac{\lambda-\mu_{j}+j-1}{\lambda+\mu_{j}+n-j+1}=-\frac{-\lambda+\mu_{j}-j+1}{\lambda+\mu_{j}+n-j+1}
$$

and $\eta_{0}(\lambda)=c(\lambda)$.

Proof. First we apply $\mathcal{C}_{m}^{\lambda-n / 2}$ to (6.2) from the left, using that $\mathcal{C}_{m}^{\lambda-n / 2}$ commutes with $\operatorname{pr}_{\sigma}$ and that $\mathcal{C}_{m}^{\lambda-n / 2} \circ \pi_{\lambda}(Y)=\pi_{-\lambda} \circ \theta(Y) \circ \mathcal{C}_{m}^{\lambda-n / 2}=-\pi_{-\lambda}(Y) \circ \mathcal{C}_{m}^{\lambda-n / 2}$. We then get:

$$
\begin{aligned}
\left(\omega(\sigma)-\omega(\mu)+2 \frac{m(n-m)}{n} \lambda\right) & \eta_{\sigma}(\lambda-n / 2) \omega_{\sigma \mu}(Y)= \\
& -\left(\omega(\sigma)-\omega(\mu)-2 \frac{m(n-m)}{n} \lambda\right) \eta_{\mu}(\lambda-n / 2) \omega_{\sigma \mu}(Y) .
\end{aligned}
$$

As $\omega_{\sigma \delta}(Y)$ is non-zero, for generic $\lambda$ it can be canceled out.

Now insert the expression from Lemma 6.1 to get

$$
\omega\left(\mu+2 \epsilon_{j}\right)-\omega(\mu)=\frac{2 m(n-m)}{n}\left(\mu_{j}+n / 2-(j-1)\right)
$$

and the claim follows. The last statement follows from the fact that $\pi_{\lambda}$ is irreducible for generic $\lambda$, hence, iterated application of (6.1) will in the end reach all $K$-types starting from the trivial $K$-type.

Lemma 6.3 tells us that the evaluation of $\eta_{\mu}(\lambda)$ can be done in two steps. First we determine the function $\eta_{0}(\lambda)$ and then use (6.3) as an inductive procedure to determine the rest. The final result is given in the following theorem. It is presented in terms of $\Gamma$-functions associated to the cone $\Omega$ of $m \times m$ positive definite matrices, namely,

$$
\Gamma_{\Omega}(\lambda)=\pi^{m(m-1) / 4} \prod_{j=1}^{m} \Gamma\left(\lambda_{j}-(j-1) / 2\right), \quad \lambda=\left(\lambda_{1}, \ldots, \lambda_{m}\right) \in \mathbb{C}^{m} .
$$


This integral is a generalization of $\Gamma_{m}(\lambda)$ in (3.5); cf. [16, p. 123], 70, Sec. 2.2]. In the following the scalar parameters, which occur in the argument of $\Gamma_{\Omega}$, are interpreted as vector valued, for instance, $n \sim(n, \ldots, n), \lambda \sim(\lambda, \ldots, \lambda)$.

Theorem $6.6(\mathbf{5 7}])$. Let $\Lambda^{+}(\mathcal{B})$ be the sublattice in Theorem 5.4 parametrizing the $L$-spherical representations of $K$, let $\mu=\left(\mu_{1}, \ldots, \mu_{m}\right) \in \Lambda^{+}(\mathcal{B})$, and $\lambda \in \mathbb{C}$. Then the $K$-spectrum of the cosine transform $\mathcal{C}_{m}^{\lambda}$ is given by:

$$
\eta_{\mu}(\lambda)=(-1)^{|\mu| / 2} \frac{\Gamma_{m}(n / 2)}{\Gamma_{m}(m / 2)} \frac{\left.\Gamma_{m}((\lambda+m) / 2)\right)}{\Gamma_{m}(-\lambda / 2)} \frac{\Gamma_{\Omega}((\mu-\lambda) / 2)}{\Gamma_{\Omega}((\lambda+n+\mu) / 2)} .
$$

REMARK 6.7. Owing to (3.12), the spectrum of the normalized cosine transform $\mathscr{C}_{m}^{\lambda}$ has the simpler form

$$
\tilde{\eta}_{\mu}(\lambda)=(-1)^{|\mu| / 2} \frac{\Gamma_{\Omega}((\mu-\lambda) / 2)}{\Gamma_{\Omega}((\lambda+n+\mu) / 2)} .
$$

In the case $m=1$ this formula coincides with (2.6).

REMARK 6.8. In Section 4 we referred to the result of Vogan and Wallach on the meromorphic continuation of the intertwining operator $J(\lambda)$. This result is not needed for the computation of $\eta_{\mu}(\lambda)$. Indeed, it is enough to know that $J(\lambda)$ is holomorphic on some open subset of $\mathbb{C}$ as that is all what is needed to determine $\eta_{\mu}(\lambda)$ in Theorem 6.6. We can then extend $\mathcal{C}_{m}^{\lambda}$ meromorphically on each $K$-type. Note, however, that this is weaker than the statement in 83] which extends $\mathcal{C}_{m}^{\lambda} f$ for all smooth functions.

\section{References}

[1] A. D. Aleksandrov. On the theory of mixed volumes of convex bodies. II. New inequalities between mixed volumes and their applications. Mat. Sbornik N.S., 2:1205-1238, 1937.

[2] S. Alesker. The $\alpha$-cosine transform and intertwining integrals on real grassmannians. 2003.

[3] S. Alesker and J. Bernstein. Range characterization of the cosine transform on higher Grassmannians. Adv. Math., 184(2):367-379, 2004.

[4] R. Askey and S. Wainger. On the behavior of special classes of ultraspherical expansions. I, II. J. Analyse Math., 15:193-220, 1965.

[5] A. Bernig. Algebraic integral geometry. arXiv:1004.3145v3, 2011.

[6] W. Blaschke. Kreis und Kugel. Chelsea Publishing Co., New York, 1949.

[7] T. Branson, G. Ólafsson, and B. Ørsted. Spectrum generating operators and intertwining operators for representations induced from a maximal parabolic subgroup. J. Funct. Anal., 135(1):163-205, 1996.

[8] J.-L. Brylinski and P. Delorme. Vecteurs distributions $H$-invariants pour les séries principales généralisées d'espaces symétriques réductifs et prolongement méromorphe d'intégrales d'Eisenstein. Invent. Math., 109(3):619-664, 1992.

[9] J.-L. Clerc. Intertwining operators for the generalized principal series on symmetric $R$-spaces. arxiv:1209.0691v1, 2012.

[10] G. van Dijk and S. C. Hille. Canonical representations related to hyperbolic spaces. J. Funct. Anal., 147(1):109-139, 1997.

[11] G. van Dijk and S. C. Hille. Maximal degenerate representations, Berezin kernels and canonical representations. In Lie groups and Lie algebras, volume 433 of Math. Appl., pages 285-298. Kluwer Acad. Publ., Dordrecht, 1998.

[12] G. van Dijk and V. F. Molchanov. Tensor products of maximal degenerate series representations of the group SL(n,R). J. Math. Pures Appl. (9), 78(1):99-119, 1999.

[13] G. van Dijk and A. Pasquale. Canonical representations of $\operatorname{Sp}(1, n)$ associated with representations of $\mathrm{Sp}(1)$. Comm. Math. Phys., 202(3):651-667, 1999.

[14] A. H. Dooley and G. Zhang. Generalized principal series representations of $\mathrm{SL}(1+n, \mathbf{C})$. Proc. Amer. Math. Soc., 125(9):2779-2787, 1997. 
[15] G. I. Eskin. Boundary value problems for elliptic pseudodifferential equations, volume 52 of Translations of Mathematical Monographs. American Mathematical Society, Providence, R.I., 1981.

[16] J. Faraut and A. Korányi. Analysis on symmetric cones. Oxford Mathematical Monographs. The Clarendon Press Oxford University Press, New York, 1994.

[17] G. B. Folland. A course in abstract harmonic analysis. Studies in Advanced Mathematics. CRC Press, Boca Raton, FL, 1995.

[18] R. L. Frank and E. H. Lieb. Inversion positivity and the sharp Hardy-Littlewood-Sobolev inequality. Calc. Var. Partial Differential Equations, 39(1-2):85-99, 2010.

[19] R. L. Frank and E. H. Lieb. Spherical reflection positivity and the Hardy-Littlewood-Sobolev inequality. concentration, functional inequalities and isoperimetry. Contemp. Math., 545:89$102,2011$.

[20] J. H. G. Fu. Algebraic integral geometry. arXiv: 1103.6256v2, 2012.

[21] A. D. Gadzhiev. Differential properties of the symbol of a singular operator in spaces of Bessel potentials on a sphere. Izv. Akad. Nauk Azerbaı̆dzhan. SSR Ser. Fiz.-Tekhn. Mat. Nauk, 3(1):134-140, 1982. in Russian.

[22] A. D. Gadzhiev. Exact theorems on multipliers of spherical expansions and some of their applications. In Special problems in function theory, No. IV (Russian), pages 73-100. "Èlm", Baku, 1989.

[23] R. J. Gardner. Geometric tomography, volume 58 of Encyclopedia of Mathematics and its Applications. Cambridge University Press, Cambridge, 1995.

[24] R. J. Gardner and A. A. Giannopoulos. p-cross-section bodies. Indiana Univ. Math. J., 48(2):593-613, 1999.

[25] S. S. Gelbart. A theory of Stiefel harmonics. Trans. Amer. Math. Soc., 192:29-50, 1974

[26] I. M. Gel'fand, M. I. Graev, and R. Roşu. The problem of integral geometry and intertwining operators for a pair of real Grassmannian manifolds. J. Operator Theory, 12(2):359-383, 1984.

[27] I. M. Gel'fand and Z. Ya. Šapiro. Homogeneous functions and their extensions. Uspehi Mat. Nauk (N.S.), 10(3(65)):3-70, 1955. in Russian.

[28] S.G. Gindikin. Analysis on homogeneous domains. Russian Math. Surveys, 19(4):1-89, 1964.

[29] P. Goodey and R. Howard. Processes of flats induced by higher-dimensional processes. Adv. Math., 80(1):92-109, 1990.

[30] P. Goodey, V. Yaskin, and M. Yaskina. Fourier transforms and the Funk-Hecke theorem in convex geometry. J. Lond. Math. Soc. (2), 80(2):388-404, 2009.

[31] E. L. Grinberg and B. Rubin. Radon inversion on Grassmannians via Gårding-Gindikin fractional integrals. Ann. of Math. (2), 159(2):783-817, 2004.

[32] H. Groemer. Geometric applications of Fourier series and spherical harmonics, volume 61 of Encyclopedia of Mathematics and its Applications. Cambridge University Press, Cambridge, 1996.

[33] Harish-Chandra. Harmonic analysis on real reductive groups iii. the Maass-Selberg relations and the Plancherel formula. Ann. of Math. (2), 104(1):117-201, 1976.

[34] S. Helgason. Differential geometry, Lie groups, and symmetric spaces, volume 80 of Pure and Applied Mathematics. Academic Press Inc., New York, 1978.

[35] S. Helgason. Groups and geometric analysis. Integral geometry, invariant differential operators, and spherical functions, volume 83 of Mathematical Surveys and Monographs. American Mathematical Society, Providence, RI, 2000.

[36] P. E. T. Jorgensen and G. Ólafsson. Unitary representations of Lie groups with reflection symmetry. J. Funct. Anal., 158(1):26-88, 1998.

[37] P. E. T. Jorgensen and G. Ólafsson. Unitary representations and Osterwalder-Schrader duality. In The mathematical legacy of Harish-Chandra (Baltimore, MD, 1998), volume 68 of Proc. Sympos. Pure Math., pages 333-401. Amer. Math. Soc., Providence, RI, 2000.

[38] M. Kanter. The $L^{p}$ norm of sums of translates of a function. Trans. Amer. Math. Soc., 79:35-47, 1973.

[39] S. P. Khèkalo. Riesz potentials in the space of rectangular matrices, and the iso-Hyugens deformation of the Cayley-Laplace operator. Dokl. Math., 63(1):35-37, 2001. Translated from Dokl. Akad. Nauk, 376(2): 168-170. 
[40] S. P. Khèkalo. The Cayley-Laplace differential operator on the space of rectangular matrices. Izv. Math., 61(1):191-219, 2005. Translated from Izv. Ross. Akad. Nauk Ser. Mat., 69:1, 195-224, 2005.

[41] S. P. Khèkalo. The Igusa zeta function associated with a complex power function on the space of rectangular matrices. Math. Notes, 78(5):719-734, 2005. Translated from Mat. Zametki, 78:5, 773-791, 2005.

[42] A. W. Knapp. Representation theory of semisimple groups, volume 36 of Princeton Mathematical Series. Princeton University Press, Princeton, NJ, 1986. An overview based on examples.

[43] A. W. Knapp and E. M. Stein. Intertwining operators for semisimple groups. Ann. of Math., 93:489-578, 1971.

[44] A. W. Knapp and E. M. Stein. Intertwining operators for semisimple groups. II. Invent. Math., 60(1):9-84, 1980.

[45] A. Koldobsky. Inverse formula for the Blaschke-Levy representation. Houston J. Math., 23(1):95-108, 1997.

[46] A. Koldobsky. Fourier analysis in convex geometry, volume 116 of Mathematical Surveys and Monographs. American Mathematical Society, Providence, RI, 2005.

[47] A. Koldobsky and H. König. Aspects of the isometric theory of Banach spaces. In Handbook of the geometry of Banach spaces, Vol. I, pages 899-939. North-Holland, Amsterdam, 2001.

[48] V. S. Kryuchkov. Differential properties of the symbol of the singular integral CalderónZygmund operator. Trudy Mat. Inst. Steklov., 170:148-160, 276, 1984. Studies in the theory of differentiable functions of several variables and its applications, X.

[49] P. Levy. Théorie de l'addition des variables aléatoires. Gauthier-Villars, 1937.

[50] E. Lutwak. Centroid bodies and dual mixed volumes. Proc. London Math. Soc. (3), 60(2):365391, 1990.

[51] G. Matheron. Un théorème d'unicité pour les hyperplans poissoniens. J. Appl. Probability, 11:184-189, 1974.

[52] S. Meda and R. Pini. Spherical convolution with kernels having singularities on an equator. Boll. Un. Mat. Ital. B (7), 5(2):275-290, 1991.

[53] K.-H. Neeb and G. Ólafsson. Reflection positivity and conformal symmetry. arXiv:1206.2039, 2012.

[54] A. Neyman. Representation of $L_{p}$-norms and isometric embedding in $L_{p}$-spaces. Israel $J$. Math., 48(2-3):129-138, 1984.

[55] G. Ólafsson. Fourier and Poisson transformation associated to a semisimple symmetric space. Invent. Math., 90(3):605-629, 1987.

[56] G. Ólafsson and A. Pasquale. On the meromorphic extension of the spherical functions on noncompactly causal symmetric spaces. J. Funct. Anal., 181(2):346-401, 2001.

[57] G. Ólafsson and A. Pasquale. The $\operatorname{Cos}^{\lambda}$ and $\operatorname{Sin}^{\lambda}$ transforms as intertwining operators between generalized principal series representations of SL(n+1, $\mathbb{K})$. Adv. Math., 229(1):267-293, 2012.

[58] E. Ournycheva and B. Rubin. Composite cosine transforms. Mathematika, 52(1-2):53-68, 2005.

[59] E. Ournycheva and B. Rubin. The composite cosine transform on the Stiefel manifold and generalized zeta integrals. In Integral geometry and tomography, volume 405 of Contemp. Math., pages 111-133. Amer. Math. Soc., Providence, RI, 2006.

[60] A. Pasquale. Maximal degenerate representations of $\operatorname{SL}(n+1, \mathbf{H})$. J. Lie Theory, 9(2):369382,1999

[61] B. A. Plamenevski1. Algebras of pseudodifferential operators, volume 43 of Mathematics and its Applications (Soviet Series). Kluwer Academic Publishers Group, Dordrecht, 1989.

[62] B. Rubin. Fractional calculus and wavelet transforms in integral geometry. Fract. Calc. Appl. Anal., 1(2):193-219, 1998.

[63] B. Rubin. Inversion of fractional integrals related to the spherical Radon transform. J. Funct. Anal., 157(2):470-487, 1998.

[64] B. Rubin. Fractional integrals and wavelet transforms associated with Blaschke-Levy representations on the sphere. Israel J. Math., 114:1-27, 1999.

[65] B. Rubin. Inversion and characterization of the hemispherical transform. J. Anal. Math., $77: 105-128,1999$. 
[66] B. Rubin. Inversion formulas for the spherical Radon transform and the generalized cosine transform. Adv. in Appl. Math., 29(3):471-497, 2002.

[67] B. Rubin. Radon, cosine and sine transforms on real hyperbolic space. Adv. Math., 170(2):206-223, 2002.

[68] B. Rubin. Notes on Radon transforms in integral geometry. Fract. Calc. Appl. Anal., 6(1):2572, 2003.

[69] B. Rubin. Intersection bodies and generalized cosine transforms. Adv. Math., 218(3):696-727, 2008.

[70] B. Rubin. Funk, cosine, and sine transforms on stiefel and grassmann manifolds. To appear in J. of Geom. Anal., 2012.

[71] B. Rubin and G. Zhang. Generalizations of the Busemann-Petty problem for sections of convex bodies. J. Funct. Anal., 213(2):473-501, 2004.

[72] W. Rudin. $L^{p}$-isometries and equimeasurability. Indiana Univ. Math. J., 25(3):215-228, 1976.

[73] S. G. Samko. Generalized Riesz potentials and hypersingular integrals with homogeneous characteristics; their symbols and inversion. Trudy Mat. Inst. Steklov., 156:157-222, 263, 1980. Studies in the theory of differentiable functions of several variables and its applications, VIII.

[74] S. G. Samko. Singular integrals over a sphere and the construction of the characteristic from the symbol. Izv. Vyssh. Uchebn. Zaved. Mat., (4):28-42, 1983.

[75] R. Schneider. Convex bodies: the Brunn-Minkowski theory, volume 44 of Encyclopedia of Mathematics and its Applications. Cambridge University Press, Cambridge, 1993.

[76] V. I. Semjanisty̌r. Some integral transformations and integral geometry in an elliptic space. Trudy Sem. Vektor. Tenzor. Anal., 12:397-441, 1963.

[77] E. Spodarev. On the rose of intersections of stationary flat processes. Adv. in Appl. Probab., 33(3):584-599, 2001

[78] E. Spodarev. Cauchy-Kubota-type integral formulae for the generalized cosine transforms. Izv. Nats. Akad. Nauk Armenii Mat., 37(1):52-69 (2003), 2002.

[79] R. S. Strichartz. Convolutions with kernels having singularities on a sphere. Trans. Amer. Math. Soc., 148:461-471, 1970

[80] R. S. Strichartz. The explicit Fourier decomposition of $L^{2}(\mathrm{SO}(n) / \mathrm{SO}(n-m))$. Canad. J. Math., 27:294-310, 1975.

[81] M. Takeuchi. Modern spherical functions, volume 135 of Translations of Mathematical Monographs. American Mathematical Society, Providence, RI, 1994. Translated from the 1975 Japanese original.

[82] T. Ton-That. Lie group representations and harmonic polynomials of a matrix variable. Trans. Amer. Math. Soc., 216:1-46, 1976.

[83] D. A. Vogan, Jr. and N. R. Wallach. Intertwining operators for real reductive groups. Adv. Math., 82(2):203-243, 1990.

[84] J. A. Wolf. Harmonic analysis on commutative spaces, volume 142 of Mathematical Surveys and Monographs. American Mathematical Society, Providence, RI, 2007.

[85] G. Zhang. Radon transform on real, complex, and quaternionic Grassmannians. Duke Math. J., 138(1):137-160, 2007.

[86] G. Zhang. Radon, cosine and sine transforms on Grassmannian manifolds. Int. Math. Res. Not. IMRN, (10):1743-1772, 2009.

Department of Mathematics, Louisiana State University, Baton Rouge, LA, 70803 USA

E-mail address: olafsson@math.lsu.edu

Laboratoire de Mathématiques et Applications de Metz (UMR CNRS 7122), UniverSité de Lorraine, 57045 Metz Cedex 1, France

E-mail address: angela.pasquale@univ-lorraine.fr

Department of Mathematics, Louisiana State University, Baton Rouge, LA, 70803 USA

E-mail address: borisr@math.lsu.edu 